\title{
Avaliação físico-química e sensorial de pão de forma elaborado com farinha de palma forrageira (Opuntia ficus-indica)
}

\author{
Physical-chemical and sensory evaluation of bread made with forage palm flour (Opuntia ficus- \\ indica) \\ Evaluación físico-química y sensorial de pan elaborado con harina de palma forrajera (Opuntia \\ ficus-indica)
}

\section{Resumo}

O estudo avaliou as características físico-químicas e sensoriais do pão de forma com adição de 0\%(FP0), 5\%(FP5), $10 \%$ (FP10) e 15\%(FP15) de farinha de palma forrageira, em substituição à farinha de trigo branca. Os pães foram submetidos a análise sensorial de 106 (cento e seis) julgadores não treinados. O pão FP5 foi o mais aceito, com um índice de aceitabilidade de 75\%. Foram realizadas análises de compostos fenólicos totais e atividade antioxidante pelo método da captura do radical ABTS •+, em FP0 e FP5, entretanto, as amostras analisadas não apresentaram, estatisticamente, diferença significativa entre si pelo teste de Tukey $(\mathrm{p}<0,05)$. Em FP5, a farinha da palma forrageira aumentou o teor de fibra e de micronutrientes, tais como cálcio $(\mathrm{Ca})$, magnésio $(\mathrm{Mg})$, potássio $(\mathrm{K})$, zinco $(\mathrm{Zn})$ e vitamina $\mathrm{C}$, sem alterar os parâmetros texturométricos $(\mathrm{p}<0,05)$. $\mathrm{O}$ pão formulado com a farinha da palma forrageira pode ser uma alternativa de baixo custo para incorporar nutrientes importantes na dieta humana, entretanto, é necessário realizar estudos sobre a presença de fatores antinutricionais. Considerando o índice de aceitabilidade alcançado pelo pão de forma, incorporação da farinha da palma forrageira possibilita o aumento de micronutrientes importantes na dieta humana em valores bem superiores aos valores encontrados no pão de forma convencional. Apesar de a palma forrageira possuir coloração verde, essa característica não ofereceu resistência à aceitação do pão de forma por parte dos potenciais consumidores do produto e pode ser atrativo para crianças na fase pré-escolar.

Palavras-chave: Palma forrageira; Pão de forma; Micronutrientes; Avaliação.

\begin{abstract}
The study evaluated the physicochemical and sensory characteristics of loaf Bread with the addition of 0\%(FP0), $5 \%$ (FP5), 10\%(FP10) and 15\%(FP15) of forage palm flour, replacing the flour of white wheat. The breads were subjected to sensory analysis by 106(one hundred and six) untrained judges. The FP5 bread was the most accepted, with na acceptability rate of $75 \%$. Analyzes of total phenolic compounds and antioxidant activity were performed by the ABTS $\bullet^{+}$radical capture method, in FP0 and FP5, however, the analyzed samples did not show statistically significant difference between them by the Tukey test $(\mathrm{p}<0.05)$. In FP5, the forage palm flour increased the contento of fiber and micronutrients, such as calcium $(\mathrm{Ca})$, magnesium $(\mathrm{Mg})$, potassium $(\mathrm{K})$, zinc $(\mathrm{Zn})$ and vitamin $\mathrm{C}$, without changing the texturometric parameters $(\mathrm{p}<0.05)$. Bread formulated with forage palm flour can be a low-cost aternative to incorporate important nutrients in the human diet, however, it is necessary to carry out studies on the presence of antinutritional factors. Considering the acceptability índex achieved by sliced Bread, incorporation of forage palm flour enables the increase of important micronutrients in the hum dieta t values much higher than the values found in conventionally baked Bread. Although the forage cactus has a green color, this characteristic did not offer resistance to the acceptance of the loaf of Bread by potential consumers of the product and may be attrative to children in the preschool phase.
\end{abstract}

Keywords: Forage palm; Loaf bread; Micronutrients; Assessment. 


\section{Resumen}

El estúdio evaluó las características fisioquímicas y sensoriales del pan de molde com la adición de 0\%(FPO), $5 \%(\mathrm{FP} 5), 10 \%(\mathrm{FP} 10)$ y 15\%(FP15) de harina de palma forrajera, en sustitución de la harina de trigo blanco. Los panes fueron sometidos a análisis sensorial por 106(ciento seis) jueces no capacitados. El pan del FP5 fue el más aceptado, con tasa de aceptabilidad del 75\%. Los análisis de compuestos fenólicos totales y actividad antioxidante se

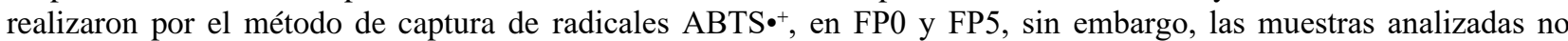
mostraron diferencia estadisticamente significativa entre ellos por prueba de Tukey ( $<<0.05)$. En el FP5, la harina de palma forrajera incrementó el contenido de fibra y micronutrientes, como calcio (Ca), magnesio $(\mathrm{Mg})$, potasio $(\mathrm{K})$, zinc $(\mathrm{Zn})$ y vitamina $\mathrm{C}$, sin alterar los parámetros texturométricos $(\mathrm{p}<0.05)$. El pan elaborado con harina de palma forrajera puede ser uma alternativa de bajo costo para incorporar nutrientes importantes en la dieta humana, sin embargo, es necesario realizar estudios sobre la presencia de factores antinutricionales. Considerando el índice de aceptabilidad alcanzado por el pan de molde, la incorporación de harina de palma forrajera permite el incremento de micronutrientes en la dieta humana em valores superiores a los valores que se encuentran en el pan de molde convencional. Si bien el cactus tiene um color verde, esta característica no ofreció resistencia a la aceptación del pan por parte de los potenciales consumidores del producto y puede resultar atractiva para los niños en la etapa preescolar. Palabras clave: Palma forrajera; Pan de molde; Micronutrientes; Evaluación.

\section{Introdução}

A busca por alternativas alimentares mais saudáveis está levando a população a explorar fontes diversas de matérias primas. Um exemplo dessas fontes alternativas é a palma forrageira. Embora no Brasil a palma tenha uso quase exclusivo para forragem, em países como o México, a palma é cultivada para alimentação humana (Urbiola et al., 2010).

A palma forrageira (Opuntia ficus-indica) é conhecida por ser uma fonte importante de compostos bioativos, como betalaínas, polifenóis, carotenóides, vitamina C e sais minerais (Utkarsha et al., 2010). Auxilia na eliminação de toxinas do álcool e do cigarro que são absorvidas pelo organismo, auxilia o organismo a metabolizar a gordura, diminuir a concentração de açúcar no sangue e, com isso, colabora na redução das taxas de colesterol e no controle da diabete. Além disso, como possui muitas fibras solúveis e insolúveis, a palma forrageira colabora para o bom funcionamento do sistema digestivo além de impedir a concentração de elementos cancerígenos (Nunes, 2011).

A adição da farinha de palma às farinhas comerciais, na proporção de 4 a $6 \%$, aumenta o conteúdo de cálcio e fibra do produto, melhorando as características nutricionais de diferentes alimentos (Villegas et al., 2010). Quando incorporada à farinha de trigo, a farinha da palma modifica os parâmetros reológicos da massa e, portanto, as propriedades físicas e sensoriais do pão. Porém, se a substituição for de até $5 \%$, as propriedades físicas e sensoriais não se alteram, aumenta o rendimento, o conteúdo fenólico total e ainda pode ser considerada como um potencial ingrediente funcional aos produtos de panificação (Msaddak et al., 2017).

Considerando que o semiárido do nordeste brasileiro oferece um clima adequado para o desenvolvimento da palma forrageira e a necessidade de aumentar a disponibilidade de micronutrientes na dieta dos indivíduos da região, esse estudo teve como objetivo a avaliação da utilização da farinha da palma forrageira (Opuntia ficus-indica) como ingrediente na formulação de pão de forma, avaliando o resultado dessa adição nas características químicas, físico-químicas, microbiológicas e sensoriais do pão.

\section{Metodologia}

A palma forrageira, com idade aproximada de 1(um) ano, foi adquirida de uma plantação localizada na cidade Piranhas-AL, Brasil (9³6’06.3” S e 3745’28,1”W). Todos os ingredientes utilizados na formulação dos pães foram adquiridos no comércio local da mesma cidade. De acordo com Pereira et al. (2018), o estudo é classificado como laboratorial com abordagem quantitativa. 


\subsection{Obtenção da farinha da palma forrageira}

Os cladódios da palma foram colhidos pela manhã, foram previamente selecionados, lavados em água corrente e escovados com escova de cerdas de plástico. A sanitização foi feita com solução de hipoclorito de sódio (200 p.p.m.) por 15(quinze) minutos e enxaguados em água corrente (Silva, et al., 2016).

Os cladódios foram cortados em cubos de aproximadamente $1 \mathrm{~cm}$, utilizando faca de aço inoxidável e, depois, espalhados em bandejas de aço inoxidável em camadas de $2 \mathrm{~cm}$ de espessura. A secagem foi realizada em estufa com circulação de ar forçado, por 24 horas a $60{ }^{\circ} \mathrm{C}$ (Castillo et al., 2013). A matéria desidratada foi moída em triturador manual, modelo B03 da BOTINI, e peneirado em peneira de aço Inox - ASTM 45-MESH/TYLER 42 - 0,355 mm, acondicionada em recipiente plásticos de cor escura e mantidos em temperatura de refrigeração.

\subsection{Elaboração dos pães de forma}

A elaboração do pão de forma foi adaptada de Canella-Rawls (2012), sendo: farinha de trigo tipo 1 - enriquecida com ferro e ácido fólico, fermento biológico seco instantâneo (2\%), ovos branco de galinha (5\%), sal refinado iodado (2\%), açúcar cristal (4\%), leite em pó integral dissolvido de acordo com as informações do fabricante (50\%) e óleo de girassol (7\%). Foi realizada a substituição da farinha de trigo branca pela farinha de palma nas proporções de: 0\% (FP0); 5\% (FP5); 10\%(FP10) e 15\%(FP15). Foi utilizada balança digital (Filizola®, Brasil) com precisão de 0,1 g e capacidade máxima de $15 \mathrm{~kg}$. Para o preparo da massa foi utilizada batedeira planetária ARNO modelo Deluxe. Os pães foram assados em forma de alumínio com revestimento antiaderente $(26 \times 10 \times 7 \mathrm{~cm})$ e em forno de fogão doméstico a gás GLP. Não foi adicionado conservante.

\subsection{Aplicação do teste sensorial}

Foi aplicado o teste de aceitação de consumidor utilizando a escala hedônica estruturada de nove pontos. Participaram do teste sensorial 106(cento e seis) julgadores não treinados. Os julgadores receberam uma bandeja com três recipientes identificados com código de três dígitos, contendo $30 \mathrm{~g}$ de FP5, FP10 e FP15. A ordem de apresentação das amostras foi balanceada e o delineamento seguiu o modelo apresentado em Kemp et al. (2009), adaptado para três amostras. Os julgadores avaliaram a aparência geral das amostras, porosidade do miolo, cor do miolo, aroma, textura na boca e sabor de cada pão (Msaddak et al., 2017). Atribuíram nota de 1 (desgostei extremamente) a 9(gostei extremamente) para os parâmetros avaliados (KEMP et al., 2009). Os julgadores também responderam com que frequência comeriam cada amostra e a intenção de compra de cada pão. Os pães foram produzidos no mesmo dia da aplicação do teste para que não perdessem as características de frescor.

Uma vez que o estudo envolveu a participação de seres humanos, a proposta foi submetida ao Comitê de Ética em Pesquisa - CEP da Fundação Universidade Federal de Sergipe - UFS, cadastrado com o Certificado de Apresentação para Apreciação Ética (CAAE) número: 00182818.5.0000.5546.

\subsection{Determinação da atividade antioxidante}

A determinação de atividade antioxidante foi realizada pelo o ensaio de (ABTS) - 2,2 AZINO BIS (3-ethylbenzo thiazoline 6 sulfonic acid) diammoninum salt PM $=548,68$ - foi realizada de acordo com o método descrito por Kuskoski et al. (2005). Essa determinação foi realizada na palma forrageira in natura, em FP0, FP5, na farinha de palma forrageira com tempo de produção igual a 0(zero) mês (FT0), 2(dois) meses (FT2) e 6(seis) meses (FT6). Para a leitura da absorbância no comprimento de onda de 734nm foi utilizado o espectrofotômetro UV (Modelo UV-2601 Rayleígh). A curva padrão de Trolox nas concentrações de 100 a $2000 \mu \mathrm{mol} / \mathrm{L}$ foi empregada para obtenção dos resultados. Todas as análises foram realizadas em triplicata e os resultados foram expressos em $\mu$ mol de Trolox equivalente (TE) / 100g. 
Os compostos fenólicos totais foram determinados de acordo com o procedimento convencional espectrofotométrico de Folin-Ciocalteu e modificado por Thaipong et al. (2006). Essa determinação foi realizada na palma forrageira in natura, em PF0, FP5, FT0, FT2 e FT6. Para a leitura da absorbância no comprimento de onda de $725 \mathrm{~nm}$ foi utilizando um espectrofotômetro (Modelo UV-2601 Rayleígh) e os resultados foram expressos em ácido gálico equivalentes (GAE mg/g de amostra). Foi construída uma curva padrão de ácido gálico (10-100 mg/L) para comparação dos resultados. Todas as análises foram realizadas em triplicata e os resultados das determinações foram expressos em mg (EAG)/100g de amostra.

\subsection{Caracterização da farinha e dos pães de forma}

Para a determinação da atividade de água nas amostras, foi utilizado o medidor Aqualab - modelo 4TEV. Para a caracterização colorimétrica foi utilizado o colorímetro modelo CR-410 (Konica Minolta, Japão). Para a determinação de proteína bruta foi utilizado o método Kjeldahl. Para as características de textura foi utilizado o texturômetro Brookfield Enginner Braseq (TexturePro CT VI.2 Build 9). Para as demais análises foi utilizada a metodologia do Instituto Adolfo Lutz (2008). Os carboidratos foram determinados por diferença. Todas as determinações foram realizadas em triplicata.

\subsection{Análises microbiológicas}

Foram realizadas as análises de Coliformes a $45{ }^{\circ} \mathrm{C}$, Staphylococus Aureus coagulase positiva e Salmonellas no Instituto Tecnológico de Pesquisa do estado de Sergipe (ITPS). Estas análises foram realizadas em amostras da farinha de palma forrageira, FP0, FP5, FP10 e FP15.

\subsection{Análise estatística dos dados}

Para verificar a normalidade dos dados obtidos no teste sensorial e nas análises físico-químicas realizadas, foi utilizado o teste de normalidade de Shapiro-Wilk. Para estatística descritiva dos dados foi utilizada a ferramenta de análise de dados da Microsoft ${ }^{\circledR}$ Excel. Para a inferência estatística dos dados não paramétricos foi utilizado o teste de Kruskal-Wallis e para os dados paramétricos, o teste de Tukey. Os testes de Kruskal-Wallis e Tukey foram realizados utilizando o software livre PAST versão 3.14 .

\section{Resultados e Discussão}

\subsection{Teste sensorial}

Com relação ao teste de aceitação, intenção de compra e aceitabilidade, FP5 se diferenciou estatisticamente $(\mathrm{p}<0,05)$ de FP10 e FP15 pelo teste de Kruskal-Wallis. Alguns julgadores afirmaram que FP15 apresentava um sabor forte e desagradável enquanto que FP5 apresentava um sabor muito bom em comparação com os pães frequentemente consumidos pelos mesmos. Uma justificativa para o apreço por FP5, por parte de alguns julgadores, pode ser o fato de já conhecerem a utilização da palma forrageira como alimento humano ou já haverem consumido a mesma em alguma receita caseira em algum momento. Msaddak et al. (2017), ao avaliar sensorialmente pães com concentração de farinha de palma de 2,5\%; 5\%; 7\% e $10 \%$, informaram que ao substituir a farinha de trigo por farinha de palma a um nível de $5 \%$ na formulação de pães, as propriedades sensoriais não se alteram. O mesmo ocorreu com Ayadi et al. (2009), quando avaliou sensorialmente bolos com frações de 5; 10; 15 e 20\% de farinha de palma em substituição a farinha de trigo, naquele estudo, o bolo com 5\% de farinha de palma também foi o mais aceito no teste sensorial. A presença de mucilagem na palma e, consequentemente, na farinha produzida para a elaboração dos pães de forma, fez alguns julgadores perceberem um aspecto "pegajoso" em FP10 e FP15. Segundo Sáenz et al. (2010), a viscosidade produzida pela farinha da palma é uma propriedade importante a ser considerada durante a formulação e preparo de alimentos contendo essa matéria prima, especialmente em alimentos líquidos. A Tabela 
1apresenta o resultado da análise sensorial realizada pelos julgadores ao avaliarem os pães FP5, FP10 e FP15 com 5, 10 e $15 \%$ de farinha de palma forrageira em substituição da farinha de trigo, respectivamente.

Tabela 1 - Resultado da análise sensorial com escala hedônica de nove pontos.

\begin{tabular}{lccc}
\hline \multirow{2}{*}{ ATRIBUTOS } & \multicolumn{3}{c}{ MÉDIA \pm DESVIO PADRÃO } \\
\cline { 2 - 4 } & $7,33^{\mathrm{b}} \pm 1,65$ & $6,32^{\mathrm{a}} \pm 1,88$ & $5,76^{\mathrm{a}} \pm 2,07$ \\
\hline Aparência geral & $7^{\mathrm{b}} \pm 1,66$ & $6,28^{\mathrm{a}} \pm 1,85$ & $5,96^{\mathrm{a}} \pm 1,67$ \\
Porosidade do miolo & $7,24^{\mathrm{b}} \pm 1,76$ & $6,08^{\mathrm{c}} \pm 2,03$ & $5,36^{\mathrm{a}} \pm 2,26$ \\
Cor do miolo & $6,2^{\mathrm{b}} \pm 2,14$ & $4,98^{\mathrm{a}} \pm 2,32$ & $4,63^{\mathrm{a}} \pm 2,47$ \\
Aroma & $6,62^{\mathrm{b}} \pm 2,02$ & $5,93^{\mathrm{a}} \pm 2,41$ & $5,46^{\mathrm{a}} \pm 2,5$ \\
Textura na boca & $6,13^{\mathrm{b}} \pm 2,54$ & $5,42^{\mathrm{c}} \pm 2,56$ & $4,35^{\mathrm{a}} \pm 2,72$ \\
Sabor & &
\end{tabular}

Letras iguais na mesma linha significam que as médias não diferem entre si pelo teste Kruskal-Wallis (p<0,05). Fonte: Autores.

Para todos os atributos avaliados apresentados na Tabela 1, FP15 apresentou menores escores em comparação com FP5 e FP10, principalmente no atributo sabor, ou seja, na substituição de $15 \%$ a palma deixou um sabor muito distinto dos pães consumidos pelos julgadores.

A Tabela 2 apresenta o resultado da aceitabilidade dos pães FP5, FP10 e FP15. De acordo com Dutcosky (2013), para um produto ser considerado com boa aceitação, deve ter um índice de aceitabilidade maior ou igual a $70 \%$.

Tabela 2 - Índice de Aceitabilidade (IA) de FP5, FP10 e FP15.

\begin{tabular}{cccc}
\hline IA (\%) & FP5 & FP10 & FP15 \\
\cline { 2 - 4 } 75,0 & 64,8 & 58,4 \\
\hline \multicolumn{4}{c}{ Fonte: Autores. }
\end{tabular}

Com o resultado apresentado na Tabela 2 observa-se que a medida que aumenta a substituição de farinha de trigo por farinha de palma, o índice de aceitabilidade do pão diminui. Alguns julgadores alegaram a presença de um sabor amargo residual em FP10 e FP15. Mulheres na faixa etária de 20 a 30 anos e homens na faixa etária de 30 a 40 anos, responderam de forma positiva com relação a intenção de compra para as três formulações de pães. Uma justificativa para esse comportamento dos julgadores, nessa faixa etária, pode ser o fato de esses julgadores terem o habito de consumir alimentos mais naturais, inclusive de pão integral. Entre os 17(dezessete) julgadores avaliados, nessa faixa etária, 11(onze) afirmaram, no questionário sócio cultural, que consumiam pão integral de uma a duas vezes ao dia.

\subsection{Características físico-químicas dos pães}

A Tabela 3 apresenta o resultado da análise dos micronutrientes de FP0 e FP5.

Tabela 3 - Composição de micronutrientes de FP0 e FP5.

\begin{tabular}{lccc}
\hline \multicolumn{1}{c}{ Componente nutricional } & FP0 & FP5 & Limite de quantificação do método \\
\hline Cálcio $(\mathrm{Ca}) \mathrm{mg} / 100 \mathrm{~g}$ & 70,10 & 201,00 & 0,068 \\
Magnésio $(\mathrm{Mg}) \mathrm{mg} / 100 \mathrm{~g}$ & 33,40 & 71,30 & 0,26 \\
Potássio $(\mathrm{K}) \mathrm{mg} / 100 \mathrm{~g}$ & 121,00 & 218,00 & 0,010 \\
Sódio $(\mathrm{Na}) \mathrm{mg} / 100 \mathrm{~g}$ & 393,00 & 457,00 & 0,044 \\
Vitamina C mg/100g & 7,50 & 10,60 & - \\
Zinco $(\mathrm{Zn}) \mathrm{mg} / 100 \mathrm{~g}$ & 1,04 & 2,15 & 0,010 \\
\hline
\end{tabular}

Fonte: Autores. 
De acordo com os resultados da Tabela 1, a presença de micronutrientes na palma forrageira fez aumentar o teor desses componentes em FP5. De acordo com Msaddak et al. (2015), a substituição de farinha de palma forrageira por farinha de trigo melhora o conteúdo de fibra alimentar, cinzas, potássio, magnésio e cálcio em biscoitos. O teor de cálcio em FP5 é 186,7\% maior que o valor encontrado em FP0. Segundo a TACO (Unicamp, 2011), os valores de cálcio para o pão de forma é de $156 \mathrm{mg} / 100 \mathrm{~g}$ e para o pão de forma com farinha de trigo integral é de $132 \mathrm{mg} / 100 \mathrm{~g}$. Assim a farinha da palma aumentou em $28,8 \%$ o teor de cálcio do pão de forma com farinha de trigo branca e em 52,3\% com a farinha de trigo integral.

Ao comparamos os teores de vitamina C de FP0 (7,50mg/100g) e FP5(10,60mg/100g), houve um aumento de 41,3\% em PF5. A TACO (Unicamp, 2011) informa que em pães de forma produzidos com farinha de trigo branca e integral, foram encontrados traços de vitamina $\mathrm{C}$.

Com relação magnésio $(\mathrm{Mg})$, potássio $(\mathrm{K})$, Sódio $(\mathrm{Na})$ e Zinco $(\mathrm{Zn})$, a taxa de aumento dos teores desses micronutrientes de FP5 em relação a FP0 foi de 113,5\%; 80,2\%; 16,3\%, 106,7\% respectivamente. A TACO (Unicamp, 2011) informa que nos pães de forma produzidos com farinha de trigo branca, os teores desses micronutrientes são $24 \mathrm{mg} / 100$ para o $\mathrm{Mg}, 65 \mathrm{mg} / 100 \mathrm{~g}$ para o K, 22mg/100g para o Na e 1,3mg/100g para o Zn. FP5 apresento mais que o dobro do teor de zinco (Zn) em comparação a FP0. Cominetti e Cozzolino (2009) informam que, dentre as funções aceitas do zinco no organismo, está a sua participação no sistema imunológico.

A Tabela 4 apresenta o resultado da análise de composição centesimal dos pães FP0 e FP5.

Tabela 4 - Composição de macronutrientes de FP0 e FP5.

\begin{tabular}{lcc}
\hline \multicolumn{1}{c}{ Componente nutricional } & FP0 & FP5 \\
\hline Atividade de água & $0,956^{\mathrm{f}} \pm 0,02$ & $0,966^{\mathrm{f}} \pm 0,009$ \\
Carboidrato $(\mathrm{g} / 100 \mathrm{~g})$ & 55,59 & 58,18 \\
Fibra Bruta $(\mathrm{g} / 100 \mathrm{~g})$ & 0,40 & 0,45 \\
Lipídios $(\mathrm{g} / 100 \mathrm{~g})$ & $3,84^{\mathrm{a}} \pm 0,03$ & $3,29^{\mathrm{a}} \pm 0,01$ \\
Proteína Bruta $(\mathrm{g} / 100 \mathrm{~g})(6,25)$ & $8,24^{\mathrm{b}} \pm 0,04$ & $7,73^{\mathrm{b}} \pm 0,18$ \\
Resíduo Mineral Fixo $(\mathrm{g} / 100 \mathrm{~g})$ & $1,86^{\mathrm{c}} \pm 0,25$ & $2,51^{\mathrm{d}} \pm 0,02$ \\
Umidade $(\mathrm{g} / 100 \mathrm{~g})$ & $30,47^{\mathrm{e}} \pm 0,75$ & $28,29^{\mathrm{e}} \pm 0,72$ \\
\hline
\end{tabular}

Letras iguais na mesma linha significam que as médias não diferem entre si pelo teste de Tukey. $(\mathrm{p}<0,05)$. Fonte: Autores.

De acordo com a Tabela 4, observa-se um maior teor de fibra bruta e de resíduo mineral fixo em FP5. A análise de fibra bruta de FP0 e FP5 revelou um aumentou de 12,5\% com relação a FP5. De acordo com Hernández et al. (2010), a palma pode ser considerada uma rica fonte de fibra solúvel nas idades mais jovens e fibra insolúvel nas idades mais avançadas da plantação. Sáenz et al. (2010), publicou os valores de fibra bruta $(6,76 \mathrm{~g} / 100 \mathrm{~g})$, fibra dietética insolúvel $(28,45 \mathrm{~g} / 100 \mathrm{~g})$ e fibra dietética solúvel $(14,54 \mathrm{~g} / 100 \mathrm{~g})$ na farinha de palma forrageira.

A Tabela 5 apresenta o resultado da análise de cor instrumental realizada no miolo de FP0, FP5, FP10 e FP15.

Tabela 5 - Coordenadas L, a, b e c para o miolo de FP0, FP5, FP10 e FP15.

\begin{tabular}{ccccc}
\hline \multirow{2}{*}{ Coordenadas } & \multicolumn{4}{c}{ Médias \pm Desvio Padrão } \\
\cline { 2 - 5 } & FP0 & FP5 & FP10 & FP15 \\
\hline L & $46,37^{\mathrm{a}} \pm 3,86$ & $39,73^{\mathrm{a}, \mathrm{b}} \pm 2,74$ & $38,57^{\mathrm{b}} \pm 2,07$ & $37,13^{\mathrm{b}} \pm 1,79$ \\
$\mathrm{a}^{*}$ & $0,97^{\mathrm{c}} \pm 0,55$ & $0,07^{\mathrm{c}} \pm 0,55$ & $1,17^{\mathrm{c}} \pm 1,07$ & $1,57^{\mathrm{c}} \pm 0,58$ \\
$\mathrm{~b}^{*}$ & $19,57^{\mathrm{d}} \pm 0,90$ & $19,57^{\mathrm{d}} \pm 1,33$ & $21,73^{\mathrm{d}} \pm 1,23$ & $21,1^{\mathrm{d}} \pm 1,14$ \\
$\mathrm{C}$ & $17,97^{\mathrm{e}} \pm 1,30$ & $20,97^{\mathrm{e}, \mathrm{f}} \pm 1,86$ & $19,43^{\mathrm{e}} \mathrm{f} \pm 2,05$ & $23,3^{\mathrm{f}} \pm 2,33$ \\
$\mathrm{H}$ & $84,9^{\mathrm{g}} \pm 4,44$ & $86,467^{\mathrm{g}} \pm 1,88$ & $88,8^{\mathrm{g}} \pm 2,5$ & $86^{\mathrm{g}} \pm 0,52$ \\
\hline
\end{tabular}

Letras iguais na mesma linha significam que as médias não diferem entre si pelo teste de Tukey $(\mathrm{p}<0,05)$. Fonte: Autores. 
Conforme os valores apresentados na Tabela 5, a medida que a fração de farinha de palma forrageira aumenta, diminui o valor da luminosidade, $L^{*}$, ou seja, o pão escurece. Entretanto, pelo teste de Tukey $(p<0,05)$, não houve diferença significativa entre FP0 e FP5 e nem entre FP10 e FP15, isso significa que não se observa mudança na luminosidade entre FP0 e FP5. Ayadi et al. (2009) atribui a diminuição dos valores de L* em FP10 e FP15 a reação de Maillard e caramelização. Com relação as coordenadas a* e b*, não houve diferença estatística pelo teste de Tukey $(\mathrm{p}<0,05)$ entre FP0, FP5, FP10 e FP15. A coordenada $\mathrm{a}^{*}$, para todos os pães, apresentou um valor médio positivo, apesar de FP10 e FP15 apresentar uma coloração visivelmente mais escura, todos os pães, de acordo com a leitura do espectrofotômetro, tenderam para o vermelho. Valores distintos foram encontrados por Ayadi et al. (2009) nos bolos produzidos com farinha de palma forrageira (Opuntia fícusindica). Em substituição a farinha de trigo em 5; 10; 15 e 20\% o autor observou valores de $\mathrm{a}^{*}$ de $-5,21$ para o bolo com 5\% de farinha de palma, -6,76 para o de 10\%; -8,43 para o de 15\%; -8,24 para o de 20\% e, para o bolo controle, o autor observou 3,98 para a coordenada cromática. Essa diferença pode ser justificada por ser produtos distintos, diferenças na coloração e granulometria da farinha de trigo utilizada e idade da palma. A coordenada cromática $b^{*}$ também apresentou uma média positiva, ou seja, uma tendência para o amarelo para todos os pães. Essa característica é confirmada com os altos valores do ângulo de tonalidade $\mathrm{H}$.

A Tabela 6 apresenta o resultado da análise de textura realizada dos pães FP0 e FP5.

Tabela 6 - Parâmetros de textura de FP0 e FP5.

\begin{tabular}{lcc}
\hline \multicolumn{1}{c}{ Parâmetros analisados } & \multicolumn{2}{c}{ Média \pm Desvio Padrão } \\
\cline { 2 - 3 } & FP0 & FP5 \\
\hline Dureza (N) & $37,4^{\mathrm{a}} \pm 11,18$ & $37,8^{\mathrm{a}} \pm 19,07$ \\
Resiliência /Resistência (Adimensional) & $0,07^{\mathrm{a}} \pm 0,006$ & $0,13^{\mathrm{a}} \pm 0,015$ \\
Coesividade/coesão (Adimensional) & $0,34^{\mathrm{a}} \pm 0,011$ & $0,44^{\mathrm{a}} \pm 0,02$ \\
Elasticidade (m) & $0,139^{\mathrm{a}} \pm 0,66$ & $0,187^{\mathrm{b}} \pm 0,73$ \\
Mastigabilidade (J.m) & $180,57^{\mathrm{a}} \pm 66,18$ & $306,23^{\mathrm{a}} \pm 152,58$ \\
\hline
\end{tabular}

Letras iguais na mesma linha significam que as médias não diferem entre si pelo teste de Tukey. (p<0,05). Fonte: Autores.

De acordo com os valores apresentados, com exceção da elasticidade, não houve diferença significativa pelo teste de Tukey $(\mathrm{p}<0,05)$ para as demais características de textura. Segundo Msaddak et al. (2017), a substituição de farinha de trigo por farinha de palma forrageira em até 5\%, não modifica as características físicas do produto.

A Tabela 7 apresenta o resultado das análises de atividade antioxidante da palma in natura, da farinha da palma recém produzida (FT0), com dois meses de armazenada (FT2) e com 6(seis) meses de armazenada (FT6).

Tabela 7 - Atividade antioxidante.

\begin{tabular}{|c|c|c|c|c|c|c|}
\hline \multicolumn{7}{|c|}{ MÉDIAS \pm DESVIO PADRÃO } \\
\hline Método & $\begin{array}{c}\text { Palma } \\
\text { forrageira } \\
\text { in natura }\end{array}$ & FP0 & FP5 & FT0 & FT2 & FT6 \\
\hline $\begin{array}{c}\text { ABTS } \\
(\mu \mathrm{mol} \mathrm{de}(\mathrm{TE}) / 100 \mathrm{~g})\end{array}$ & $177,27 \pm 11,42$ & $195,56^{\mathrm{d}} \pm 12,47$ & $190,4^{\mathrm{d}} \pm 4,24$ & $40,71^{\mathrm{e}} \pm 3,65$ & $25,74^{\mathrm{f}} \pm 0,82$ & $31,11^{g} \pm 2,10$ \\
\hline $\begin{array}{c}\text { Compostos fenólicos totais } \\
(\mathrm{mg} \text { EAG } / 100 \mathrm{~g})\end{array}$ & $40,24 \pm 1,66$ & $39,96^{\mathrm{b}} \pm 1,78$ & $36,31^{b} \pm 2,24$ & $4,86^{c} \pm 0,46$ & $4,25^{\mathrm{c}} \pm 0,67$ & $4,48^{\mathrm{c}} \pm 0,20$ \\
\hline
\end{tabular}

Letras iguais na mesma linha significam que as médias não diferem entre si pelo teste de Tukey. (p<0,05). Fonte: Autores. 
A palma forrageira in natura e a farinha obtida apresentaram atividade antioxidante, entretanto, não houve diferença significativa pelo teste de Tukey $(\mathrm{p}<0,05)$ entre FP0 e FP5, tanto com relação a quantidade de compostos fenólicos totais, quanto em relação a atividade antioxidante pelo método da captura do radical ABTS ${ }^{+}$. Na formulação dos pães foi utilizado óleo de girassol que, de acordo com o fabricante, contém tocoferol e ácido ascórbico, que são substâncias antioxidantes. Pedrosa et al. (2000) relatou a presença dos mesmos componentes nas sementes de girassol e Msaddak et al. (2017), publicou que os cladódios da palma forrageira é uma importante fonte desses compostos. Com base nessas informações, é possível justificar porque não houve diferença significativa pelo teste de Tukey $(p<0,05)$ entre FP0 e FP5 com relação aos compostos fenólicos totais. Muito embora, no mesmo estudo publicado por Msaddak et al. (2017), os autores informaram que a farinha da palma forrageira melhorou o conteúdo fenólico e o potencial antioxidante do pão quando substituiu em $5 \%$ a farinha de trigo, muito embora, no presente estudo, esse fato não ocorreu.

Para a farinha da palma forrageira, tanto para o resultado dos compostos fenólicos totais, quanto para a captura do radical ABTS $\bullet^{+}$, observa-se uma queda nos valores a medida que o tempo passa, uma justificativa para esse ocorrido é que, pode ter ocorrido uma degradação destes componentes ativos apesar do armazenamento da farinha ter sido em recipiente escuro e sob refrigeração. Entretanto, o moinho que foi utilizado para produzida a farinha da palma, pode ter liberado íon ferro, uma vez que o moinho utilizado é de ferro fundido e, de acordo com Souza (2001), íons metálicos como ferro, cobalto, cobre e molibdênio podem atuar como catalisadores da decomposição de peróxidos, diminuindo assim, a presença de substâncias antioxidantes no alimento.

\subsection{Análises microbiológicas}

Com relação as análises microbiológicas realizadas em amostras de FP0, FP5, FP10, FP15 e a farinha de Palma forrageira, as amostras atenderam aos limites estabelecidos pela Instrução Normativa no 12 de 04/09/2003, do Ministério da Agricultura e a Resolução - RDC n ${ }^{\circ} 12$ de 2001 da ANVISA, com relação as análises de Coliformes a $45{ }^{\circ} \mathrm{C}$, Staphylococus Aureus coagulase positiva e Salmonellas.

\section{Considerações Finais}

O pão formulado com a farinha da palma forrageira pode ser uma alternativa de baixo custo para incorporar nutrientes importantes na dieta humana, entretanto, é necessário realizar estudos sobre a presença de fatores antinutricionais.

Considerando o índice de aceitabilidade alcançado pelo pão de forma, incorporação da farinha da palma forrageira possibilita o aumento de micronutrientes importantes na dieta humana, tais como: cálcio, magnésio, potássio e vitamina $\mathrm{C}$, em valores bem superiores aos valores encontrados no pão de forma convencional. Apesar de a palma forrageira possuir coloração verde, essa característica não ofereceu resistência à aceitação do pão de forma por parte dos potenciais consumidores do produto e pode ser atrativo para crianças na fase pré-escolar.

É importante, para estudos futuros, avaliar a atividade antimicrobiana da farinha da palma sobre os produtos obtidos a partir dela.

\section{Agradecimentos}

Agradecemos a Universidade Federal de Sergipe e ao Instituto Federal de Alagoas, pela disponibilidade da infraestrutura, reagentes e equipamentos utilizados nesse estudo. Aos técnicos dos laboratórios das instituições citadas e aos julgadores que gentilmente, participaram do teste sensorial. 


\section{Referências}

Abdelmaksoud, W., Ennouri, M. \& Attia, H. (2009). Cladodes from Opuntia fícus-indica as a source of dietary fiber: effect on dough characteristics and cake making. Industrial Crops \& Products, 30(1), 40-47.

Canella-Rawls, S. (2012). Pão: arte e ciência. (5a. ed), Editora Senac. 348p.

Castillo, O, S. F., Estrada, L., Margalef, M.I. \& Tóffoli, S. L. (2013). Obtención de harina de nopal y formulación de alfajores de alto contenido en fibra. Diaeta. (B.Aires), 31(1), 20-26.

Cominetti, C. \& Cozzolino, S.M.F. (2009). Funções Plenamente Reconhecidas de Nutrientes: Zinco. (v.07). ILSI Brasil.

Doutcosky, S. D. (2013). Análise sensorial de alimentos. (4a. ed.). Champagnat. 531p.

Fennema, O. R., Damodaran, S. \& Parkin, K.L. (2010). Química de Alimentos de Fennema. (4a. ed), Artmed. 900p.

Hernández, U. M. I., Contreras, P. M., Pérez,T. E., Hernández, Q.G., Rojas, M.J.I, Cortes, M.E. \& Rodríguez, G.M.E. (2010). Study of nutritional composition of nopal (Opuntia ficus-indica cv. Redonda) at different maturity stages. The open Nutrrition Journal, 4(1),11-16.

Instituto Adolfo Lutz. (2008). Métodos físico-químicos para análise de alimentos. Normas analíticas do Instituto Adolfo Lutz. São Paulo, 1020p.

Kemp. S. E., Hollowood. T. \& Hort, J. (2009). Sensory Evaluation: A Practical handbook. Oxford :Wiley-Blackwell. 196p.

Kuskoski, E. M., Asuero, A. G., Troncoso, A. M., Mancini-Filho, J. \& Fett, R. (2005). Aplicatíon de diversos métodos químicos para determinar actividad antioxidante en pulpa de frutos. Ciência e Tecnologia de Alimentos, 25(4), 726-732.

Msaddak, L., Abdelhedi, O., Kridene, A., Rateb, M., Belbahri, L., Ammar, E., Nasri, M. \& Zouari, N. (2017). Opuntia ficus-indica cladodes as a functional ingredient: bioactive compounds profile and their effect on antioxidant quality of bread. Lipids in Health and Disease, $16(1), 32$.

Nunes, C. dos S. (2011). Usos e aplicações da palma forrageira como uma grande fonte de economia para o semiárido nordestino. Revista Verde, 6(1),58-66.

Pedrosa, M. M., Muzquiz, M., Garcia, V. C., Burbano, C., Cuadrado, C. \& Ayet, G. (2000). Determination of caffeic and chlorogenic acids and their derivatives in different sunflower seeds. Journal of the Science of Food and Agriculture, 80(1), 459-64.

Pereira, A. S., Shitsuka, D. M., Parreira, F. J. \& Shitsuka, R. (2018). Metodologia da pesquisa científica. [e-book]. Santa Maria. Ed. UAB/NTE/UFSM. https://repositorio.ufsm.br/bitstream/handle/1/15824/Lic_Computacao_Metodologia-Pesquisa-Cientifica.pdf?sequence=1 .

Sáenz, C., Berger, H., García, J.C., Galleti, L., Cortázar, V.G. de., Higuera, I., Mondragón, C., Rodríguez Felix, A., Sepúlvera, E. \& Varnero, M.T. (2006). Utilización agroindustrial del nopal. (1a. ed). Organización de Las Naciones Unidas para la Agricultura y la Alimentación.(cap. 1), 1-6.

Sáenz, C., Sepúlveda E., Pak, N. \& Lecaros, M. (2010). Chemical and physical characterization of cactus cladode (Opuntia fícus-indica) powder. Italian Journal of Food Science, 22(4),416-422.

Souza, T.C. (2001). Alimentos: propriedades físico-químicas. (2a. ed). Cultura Médica. 204p.

Silva, W. L., Medeiros, R. A. B. D., Pires, E. \& Freitas, M.(2016). Eficiência do cloro para sanitização de hortaliças. Higiene Alimentar, 30( 256/257),132136.

Tabela brasileira de composição de alimentos / NEPA - UNICAMP. (4a ed.), (2011). NEPA- UNICAMP. 161 p.

Thaipong, K., Boonprakob, U., Crosby, K., Cisneros-Zevallos, L. \& Byrne, D.H. (2006). Comparison of ABTS, DPPH, FRAP, and ORAC assays for estimating antioxidant activity from guava fruit extracts. Journal of Food Composition and Analysis, 19(6-7), 669-675.

Urbiola, M.I.H., Padilla, M.C., Torrero, E.P., Quevedo, G.H., Molina, J. I.R., Cortes, M. E. \& García, M. E.R. (2010). Study of nutricional composition of nopal (Opuntia ficus indica cv. Redonda) at different maturity stages. The Open Nutrition Journal, 4(1), 11-16.

Utkarsha, U, Shedbalkar, S, Adki, V, Jadhav, P. \& Vishwas, A.B.(2010). Opuntia and Other Cacti: Applications and Biotechnological Insights. Tropical Plant Biol, 3(3), 136-150.

Villegas, M. A. C., Osorio, A. A. C., Molina, I. R., Cortez, E. G., Quiroga, M. A., Gaytán, N, M., Herrera, G. \& García, M. E. R. (2010). Study of the physicochemical and pasting properties of instant corn flour added with calcium and fibers from nopal powder. Journal of Food Engineering, 96(1), 401-409. 